Gardner (1953) and Kriss et al. (1955) reported certain sideeffects after administration of cobalt even for short periods. These effects included nausea, vomiting, damage to the eighth cranial nerve nuclei, thyroid hyperplasia and myxoedema, and congestive heart failure (Goodman and Gillman, 1965).

The case described above showed some of these toxic effects. The central nervous system was severely affected ; the vestibular and cochlear parts of the eighth nerve were damaged, as was shown by the abnormal caloric tests and audiometric curves. Paraesthesiae in the limbs, absent tendon jerks, and calf tenderness indicated that the patient also had a peripheral neuritis. The difficulty which she experienced in walking was probably due to a combination of vestibular damage and the peripheral neuritis.

In the series published by Kriss et al. (1955) cobalt chloride was given to children with sickle-cell anaemia, and it was found that cobalt depressed the uptake of iodine by the thyroid gland and caused thyroid hyperplasia. In the above case the iodine uptake was greatly increased. This test, however, was carried out three weeks after withdrawal of cobalt chloride, during the rebound recovery phase after thyroid suppression. The exact lesion in the thyroid has not been elicited. However, Kriss et al. cite evidence that cobalt chloride inhibits the tyrosine iodinase system, which catalyses the iodinization of tyrosine to form monoiodotyrosine. This view is strengthened by the fact that in the case reported here potassium perchlorate caused an immediate discharge of radioactive iodine from the gland, indicating that much of the iodine was still in the inorganic form.

Holly (1955) stated that cobalt is excreted primarily by the kidney, and he showed that rats receiving high doses of intraperitoneal cobalt developed renal tubular necrosis, though no glomerular damage was noted. There appear to be no previous reports of renal toxicity in man. The above patient had evidence of tubular damage, as was shown by the temporary glycosuria and the amino-aciduria. The chromatographic pattern of the amino-acids was very similar to that produced by other heavy metals-for example, copper in Kinnier Wilson's disease. There is no specific pattern of amino-aciduria caused by the different metals, and the basic lesion is agan probably due to enzyme inhibition, perhaps the phosphorylation mechanism which is important in the active reabsorption of amino-acids. It was proved, by the disappearance of the tubular lesion three months after stopping the drug, that this damage was not due to the underlying kudney disease but to the cobait administered.

In the above case it appears that the effect of cobalt on the body is of a temporary nature. Indeed it was gratifying to find that the effects on the thyroid gland, central nervous system, and renal tubules were completely reversible after the drug was stopped. The underlying condition of her renal failure, however, did not improve. The reason that this patient suffered so severely from the toxic effects of cobalt may be the combination of prolonged time of administration of the drug and the ponr underlying renal clearance.

From this case and from previous reports it appears that cobalt chloride is very effective in raising the level of haemoglobin in refractory anaemias, especially that of renal failure. This asset of the drug, however, seems to be outweighed by the widespread side-effects which it can produce. Therefore, ther 8 peutically, cobalt chloride seems to have no place in modern medicine.

I wish to thank Dr. J. R. Nassim for permission to publish details of this case.

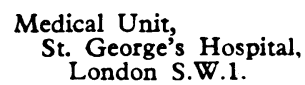

U. O. E. SCHIRRMACHER, M.B., B.S

REFERENCES

Gardner, F. H. (1953). 7. Lab. clin. Med.. 41, 56. Goodman, L. S., and Gillman, A. (1965). The Pharmacological Basis of Therapeutics, 3rd ed., p. 1406. New York.

Holly, R. G. (1955). ₹. Amer. med. Ass., 158, 1349.

Kriss, J. P., Carnes, W. H., and Gross, R. T. (1955). Y. Amer. med. Ass. 157,117 .

Weissbecker, L. (1950). Med. Mschr., Suppl. No. 9.

\section{Prevention of Regurgitation During Induction of Anaesthesia with a Cuffed Oesophageal Catheter}

Brit. med. F., 1967, 1, 545-546

The importance of preventing regurgitation of stomach contents during induction of anaesthesia, particularly in operative obstetrics and emergency general surgery, is appreciated by anaesthetists. Endotracheal intubation with a cuffed tube is still the safest method for protecting the pulmonary system from the hazards of aspiration.

Sellick ${ }^{1}$ summarized four approaches to the problem of cuffed endotracheal intubation in emergency surgery, when an empty stomach is but a wishful thought. These techniques are as follows: (1) Intubation under local anaesthesia if intubation is expected to be difficult ; Sellick suggested preliminary tracheostomy if severe maxillo-facial injuries are present. (2) Postural drainage of the pharynx in the lateral or Trendelenburg position during inhalation induction, where gravity and good suction assist in getting rid of any regurgitated contents in the pharynx, until the cuffed endotracheal tube has been passed. (3) Postural retention of gastric contents by inducing anaesthesia in the sitting position or 30 degrees head-up posture. Such technique requires rapid induction with barbiturate and muscle relaxant to achieve passage of the endotracheal tube without much delay. (4) Temporary oesophageal occlusion by means of cricoid pressure during the induction-intubation period.2 Control of active vomiting by cricoid pressure is contraindicated, since obstruction to forcible vomiting might damage the oesophagus.

A further method, based on oesophageal occlusion by a balloon catheter to control regurgitation of gastrointestinal contents during the critical induction-intubation period, is described below. This method also prevents inflation of the stomach (a potent cause of regurgitation) during the positivepressure ventilation preceding intubation with the aid of muscle relaxant. The wide-bore catheter acts as a safety-valve against any build-up of high pressure in the oesophagus behind the balloon, should active vomiting occur.

\section{METHOD}

Foley's haemostatic catheter is used-the type which acts as a self-retaining urethral catheter after prostatic operation. This has a strong balloon of 30-100 ml. capacity. The catheter, in variable sizes, has two terminal bores. A No. 26 F.G.S. is suitable for an adult patient. Analgesia of the mouth and pharynx facilitates insertion of the catheter into the oesophagus in the conscious patient without difficulty. In most cases this is not needed. The catheter is passed down the oesophagus as 
far as it will go. In most cases it passes the cardia and can be used as a stomach-tube to aspirate the stomach contents. Just before induction of anaesthesia the balloon is inflated. Once the endotracheal tube is in place and its cuff inflated, there is no need to keep the balloon inflated, but the catheter can be used for suction if it is pushed to enter the cardia.

An intravenous barbiturate and muscle-relaxant technique allows rapid and easy intubation. In cases with marked shock or in those who are seriously ill, inhalational induction is preferred, to avoid the use of intravenous thiopentone. However, once the patient is unconscious a short-acting muscle relaxant permits an easy and rapid intubation and avoids anoxic episodes due to any straining.

\section{RESULTS}

This technique has been used in the State Medical Hospitals, Qatar, during the past two years in 147 emergency cases. Local anaesthetic spray of the mouth cavity and pharynx was carried out in 25 uncooperative women undergoing obstetric intervention; the remainder tolerated the tube without any trouble.

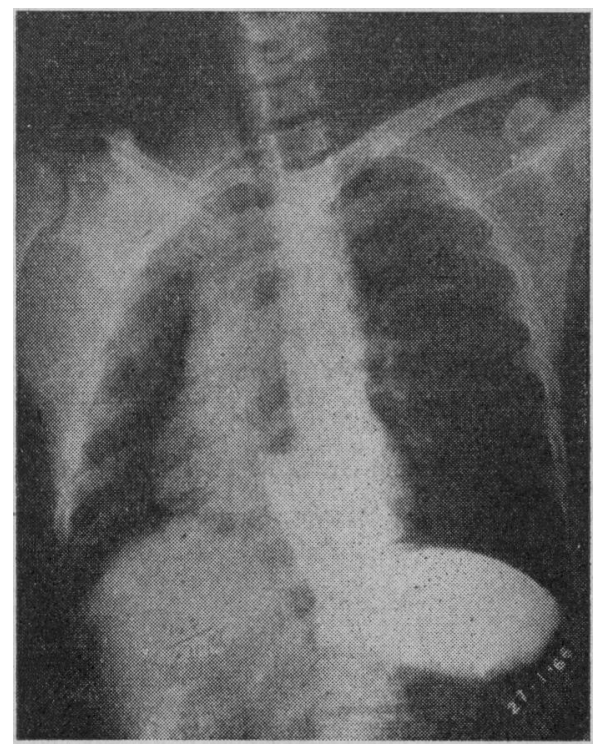

FIG. 1.-X-ray film of patient.

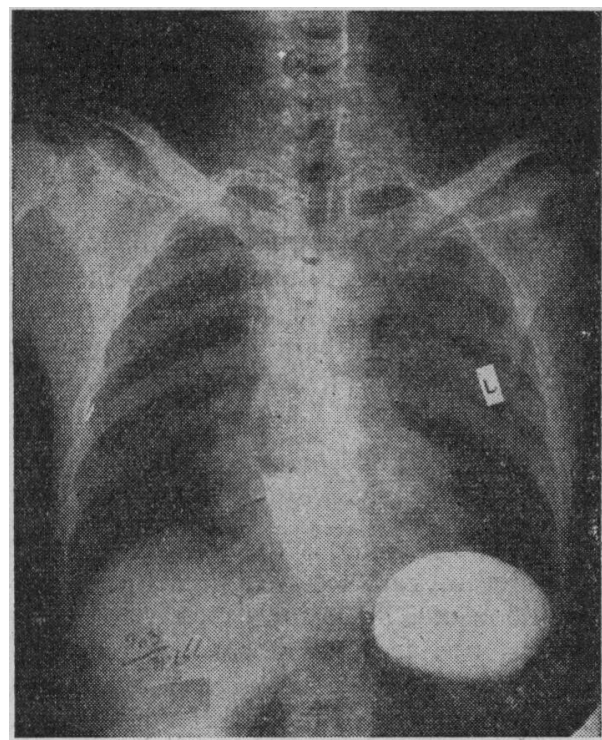

Fig. 2.- $X$-ray film taken 30 minutes after the film in Fig. 1.
Regurgitation of stomach contents was well controlled behind the ballooned oesophageal catheter in all cases. The critical induction-intubation period was safe and dry in all cases without any effort on the part of the anaesthetist. The widebore oesophageal catheter effectively drained regurgitant material behind the balloon by way of the mouth. Fig. 1 is an $x$-ray film of a patient who was given $100 \mathrm{ml}$. of liquid barium emulsion to drink. An oesophageal balloon catheter was inserted and anaesthesia with barbiturate and muscle relaxant induced. An $x$-ray film was taken with the patient in a deep head-down tilt to facilitate regurgitation of stomach contents. It reveals effective control of the opaque material behind the oesophageal cuff. Fig. 2 shows the patient in the same position 30 minutes after the first film. No leakage can be seen around the cuff. There is a hold-up of barium at the cardia, showing its efficiency as a sphincter/valve mechanism.

\section{Discussion}

Since Mendelson ${ }^{3}$ called attention to the entity of aspiration pneumonia (Mendelson syndrome) after the inhalation of acid gastric contents during anaesthesia, several methods have been employed to guard against this complication. When difficulties with endotracheal intubation are expected, intubation of the conscious patient with a cuffed tube is the safest method. ${ }^{4}$ Postural drainage of the pharynx in the lateral or supine position with a head-down tilt to facilitate the flow of pharyngeal contents away from the larynx has its advocates. ${ }^{5}$ However, any straining during the inhalational induction predisposes to regurgitation, and serious anoxic episodes may follow. The use of muscle relaxant in such positions is not wise until endotracheal intubation is completed, as the risk of gastric reflux is high after oesophageal relaxation. ${ }^{2}$

Postural retention of gastric contents by inducing anaesthesia in the sitting or 30 degrees head-up posture has become widely adopted. $^{67}$ It has been suggessted ${ }^{2}$ that induction in that position with intravenous barbiturate and muscle relaxant is hazardous, and this has been confirmed. ${ }^{5}$ This position cannot prevent but rather facilitates the entry of stomach contents into the lung if active vomiting takes place in the brief interval between loss of consciousness and onset of muscle relaxation. ${ }^{8}$ Rapid induction in the sitting position predisposes to circulatory collapse, especially in seriously ill or shocked patients. ${ }^{2}$

To prevent regurgitation, occlusion of the oesophagus by backward pressure on the cricoid has been recommended during induction of anaesthesia. ${ }^{2}$ This method requires a trained assistant to carry it out and must not be used during active vomiting, as it may lead to oesophageal rupture.

The advantages of the technique using an oesophageal catheter are its effectiveness without much effort from either the patient or the anaesthetist. The method does not need unfavourable positions. It also permits ventilation of the lungs by positive pressure without inflation of the stomach, which is a potent cause of regurgitation. The wide-bore catheter can be used to aspirate any oesophago-gastric material before, during, and after anaesthesia.

A. F. M. ZOHAIRY, M.D.

State Medical Services,

Doha, Qatar.

REFERENCES

1 Sellick, B. A., First European Congress of Anaesthesiology, Vienna, 1962 .

Lancet, 1961, 2, 404.

3 Mendelson, C. L., Amer. 尹. Obstet. Gynec., 1946, 52, 191.

- Wycoff, C. C., Anesth. Analg. Curr. Res., 1959, 38, 5

- Bourne, J. G., Anaesthesia, 1962, 17,. 379.

Edwards, G., Morton, H. J. V., Pask, E. A., and Wylie, W. D., ibid., 1956, 11, 194.

' Snow, R. G., and Nunn, J. F., Brit. F. Anaesth., 1959, 31, 493.

- O'Mullane, E. J., Lancet, 1954, 1, 1209. 